\title{
Fluctuations in Natural Populations of the Leaf Miner (Coelaenomenodera lameensis) in Relation to Different Origins of Oil Palm (Elaeis sp.) in South Benin
}

\author{
A. Coffi ${ }^{1}$, R. Philippe ${ }^{2}$, I. Glitho ${ }^{3}$ \\ ${ }^{1}$ Centre de Recherches Agricoles Plantes Pérennes (CRA-PP), Institut National de Recherches Agricoles du Bénin (INRAB), Pobè, \\ Bénin; ${ }^{2}$ Centre International de Recherche Agronomique pour le Développement, Montpellier, France; ${ }^{3}$ Laboratoire d'Entomologie \\ Appliquée, Faculté des Sciences, Université de Lomé, Lomé, Togo. \\ Email: alassane.coffi@gmx.fr
}

Received June $25^{\text {th }}, 2013$; revised July $26^{\text {th }}, 2013$; accepted August $13^{\text {th }}, 2013$

Copyright (C) 2013 A. Coffi et al. This is an open access article distributed under the Creative Commons Attribution License, which permits unrestricted use, distribution, and reproduction in any medium, provided the original work is properly cited.

\begin{abstract}
Preferences of different oil palm crosses in the station of Pobè in Benin were tested on Coelaenomenodera lameensis by monitoring the natural population for 7 consecutive years. Experiments monitoring of developmental stages of the insect were performed on material types Elaeis guineensis and Elaeis oleifera. Observations were performed every two weeks on leaves of different ranks $(25,17$ or 9$)$ depending on the degree of defoliation by counting the different stages of insect development on leaflets. The results showed that the evolution of pest density is a function of species and the origin of the materials being compared. Population densities of different stages of $C$. lameensis were more abundant in the crosses from Yocoboue where average population indices were higher at all stages of development from early observations. By contrast, in crosses La Mé, Yangambi and Deli, the numbers of larvae, pupae and adults were lower. Among these three crosses infestation was more abundant in La Mé, followed by Yangambi and Deli. E. oleifera is much less or not attacked. The stages of development (larvae and adults) were also differently held in oil palm origins in comparison. The larval population is significantly important during the short rainy season and lesser important during the dry season. These results may contribute to the development of suitable materials for the genetic improvement of breeding tolerant oil palm material to leaf miner.
\end{abstract}

Keywords: Coelaenomenodera lameensis; Leaf Miner; Oil Palm; Elaeis spp

\section{Introduction}

Oil palm, Elaeis spp. is a perennial plant native to Africa. The genus Elaeis contains three species Elaeis guineensis jacq from Africa (most common), Elaeis oleifera (HBK) Cortes of Brazilian origin and Elaeis madagascariensis Beccari met in Madagascar. The species guineensis, the most common, is grown for its fruit and almonds which provide a popular vegetable oil used in the diet for $80 \%$, oleochemicals for $19 \%$ and biodiesel for $1 \%$ [1]. The species E. oleifera, compared to its African parent E. guineensis, usually grown in the tropics, is rare and is naturally distributed in Central America, Honduras to Colombia, and in the Amazon region. It has very interesting features for genetic improvement, including the quality of the oil and the slow growth rate. Oil palm is the target of many insect pests including Coelaenomenodera lameensis Berti and Mariau (Coleoptera: Chrysomelidae-
Hispinae), commonly known as leaf miner which is very harmful in West Africa [2]. Among the many Hispinae widely distributed in the tropics, the genus Coelaenomenodera has thirty eight species including two C. elaeidis and $C$. lameensis previously known as C. Elaeidis Maulik and after C. minuta Uhmannby [3]. The distribution of C. lameensis covers Central and West Africa (Benin, Cameroon, Côte d'Ivoire, Ghana, Nigeria and Sierra Leone) [4]. It grows inside tunnels to the underside of the leaves of oil palm. The species C. elaeidis frequently develops mixed with $C$. lameensis, but the strength of its populations remains very low. These two species are parasitized by the same species of insects. The adult female lays eggs at the bottom of a small furrow dug to the underside of the leaflets parallel to the rib. They are fixed to the upper epidermis that dries here. Eggs are incompletely enveloped by a shell ocher, and covered with a 
fibrous agglomerate nature vegetable emboss marking the location of spawning. The total cycle time is 94 days (3 months) and the different stages are as follows: eggs (20.5 days), larvae (44 days: L1-11 days, L2-9 days, 9 days and L3-L4-15 days), pupa (12 days), adult gallery in 1 - 2 days and adult on the underside of leaves and 17 days before spawning $[5,6]$. During outbreaks, C. lameensis causes severe defoliation which can cause up to about $50 \%$ loss of production for two years [6-8]. The adult consumes the leaf parenchyma to the underside of the leaflets by longitudinal grooves tens of millimeters parallel to the ribs. These grooves are 12 to $15 \mathrm{~mm}$ in length and are drilled through the entire thickness of the leaflet. The most harmful damage caused by larvae in rate of 4 to 6 galleries per leaflet dries the whole palm starting with the lower palm leaf crown. The damage caused by the larvae is the most damaging because of the number and size of mines per leaflet [9]. The oleifera appears less attacked than the species guineensis $[10,11]$. Differences in sensitivity were also observed in guineensis species' [11,12]. In 2002, the plantations of the research station of Pobè suffered severe pressure due to overgrowth of C. lameensis which caused severe defoliation of palm trees [8-13]. Preliminary observations made in the field indicated that the level of infestation varied with the genetic origins of oil palm [8]. The impact of the genetic variability of oil palm on the attack of C. lameensis which is of great interest for the selection of breeding resistant or vulnerable species has been addressed [11]. According to [14], the larvae out of their galleries are less sensitive to temperature variations than eggs. However, young galleries can dry with high temperatures and this happens mainly on leaves in the top of crown. Similarly heavy rain can destroy galleries of all sizes. These conditions with the endogenous mortality participate in large part, in the reduction of populations of $C$. lameensis.

This research aims at studying the level of infestation in natural populations of $C$. lameensis on different plant materials of oil palm.

\section{Material and Methods}

The experiments were conducted under natural conditions in southern Benin on the station "Centre de Recherches Agricoles Plantes Pérennes de Pobè" ( $7^{\circ}$ North and $2^{\circ} 40$ East). The average annual rainfall in Pobè is $1200 \mathrm{~mm}$. The months of March to August are marked by the long rainy season before a short dry season until mid-September. A short rainy season occurs from September to November and a long dry season from November to March. The oil palm aged around of 8 years old was our studied material. Four (04) origins of E. guineensis (La Mé, Yangambi, Deli and Yocoboué) and two
(02) origins of E. oleifera (Brazil and Central America) were concerned in the study of fluctuations of natural populations of the pest. The experimental design is a randomized complete block. The observations were made on two palms on a line (one at the east and one at the west) and all five lines, the crossings of the 4 origins of $E$. guineensis and two origins of E. oleifera and all five lines, a total of 24 trees. The choice of trees is made randomly outside border lines for each original. On each oil palm sampled, observations were made on a palm cut at the 9th, 17th or 25th leaf depending on the level of leaf defoliation foliage. The counts were performed every 15 days. The counting of larvae, pupa and imago of $C$. lameensis occurs visually on all leaflets of the palm when the larval population level is low (average larvae per palm between 0 and 40). In the case of a high population (average greater than 40 palm larvae), the count was made on a leaflet out of 10 on a side of the palm and then to the entire palm. The bimonthly samples of leaflets for observation were scaled from January 2005 to December 2011.

The monthly rainfall is recorded during this period. The General Linear Model (GLM) was used to perform ANOVA with SAS software (SAS Institute, 1997 version 9.2). Variance analysis focused on the total number of eggs laid per year, depending on season and the series, the plant material and sampling by Poisson regression [15].

\section{Results}

\subsection{Fluctuation of Larval Populations of $C$. lameensis on Different Material}

The fluctuation of larval populations from January 2005 to July 2011 (Figure 1) showed a higher number of crossings of origin Yocoboué with 3 distinct medium peaks of around 1600, 3000 and 1900 individuals per tree respectively in 2005, 2008 and 2011. Indeed, we have witnessed a rapid and abrupt decline (between July 2006 and August 2008), and a biological level between August 2005 and June 2006 from which we observed a sawtooth evolution during the remainder of the period. Concerning oil palm from La Mé, we noticed the same trend for larval populations that have a medium biological growth (in 2005, 2008, 2010 and 2011), a biological level (between October 2005 and June 2006), and changesin sawtoothhand biological peaks. As for Yangambi and Deliorigins, we noted a biological level between August 2005 and September 2006, a biological peak of 925 for Yangambi and 650 for Deli respectively in November 2009 and July 2011. By cons, the proliferation of larval populations remained very low on E. oleifera compared to other materials. This material showed some resistance against the attack of this insect. Thus, periodic surveys have shown 


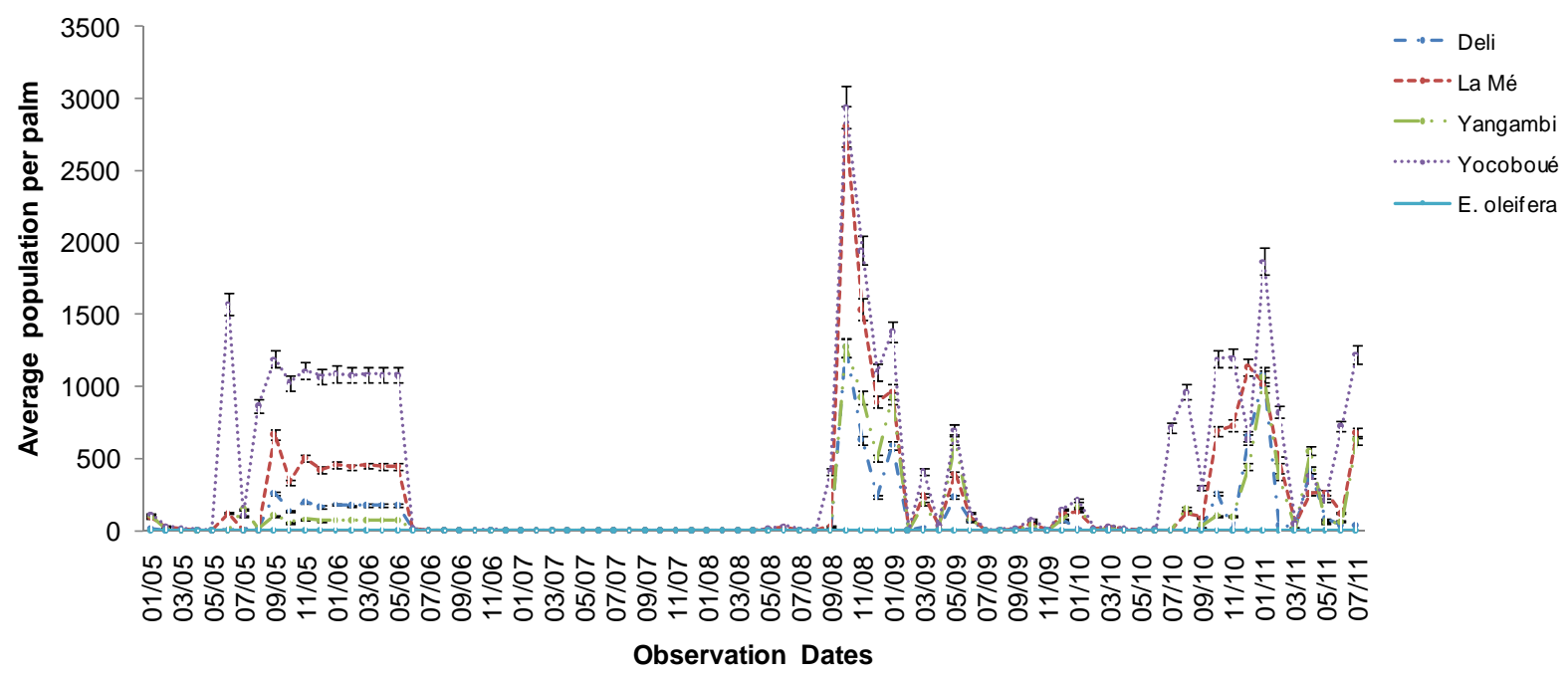

Figure 1. Dynamics of larval populations of C. lameensis from 2005 to 2011.

a large number of palm trees from Yocoboué while on the palm of the other three sources, the number of adults was very low, especially those of La Mé origin that was also sensitive compared to Yangambi and Deli. Finally, E. oleifera occupied the last position due to their nature of resistance to $C$. lameensis.

\subsection{Fluctuation of Pupae's Populations of $C$. lameensis on Different Materials}

The evolution of the pupal stage (Figure 2) was virtually identical to that of larvae in the crosses from Yocoboué and La Mé, with periods of low densities (between January and September 2005, between November 2005 and September 2008 and February and December 2009), 6 successive peaks (in September 2005, October 2008, January, April and June 2010 and finally in March 2011) and two biological levels (between October 2010 and February 2011 and between April and July 2011) were recorded. Concerning the crosses native to Yangambi, three biological peaks were observed (October 2005 and 2008 and June 2011). This trend was less noticeable on the Deli material where populations' trend was in sawtooth especially between January and July 2011. Nymphal populations remained absent on the types Oleifera during the whole period of observations.

\subsection{Fluctuation of Populations of Adults of $C$. lameensis on Different Material}

Like the nymphs, the adult populations of $C$. lameensis also varied over time (Figure 3).

On the crosses native to Yocoboué, an outbreak was characterized by three main situations: a small population (January to September 2005, November 2005 to September 2008 and March 2009 to May 2010); 4 biological peaks (October 2005 and 2008, March 2009 and August 2010) and a biological level between October 2010 and February 2011. The trend was the same in La Mé origins with the same periods of scarcity and proliferation of adult insects. To this is added a biological level between October 2010 and February 2011. On Deli and Yangambi the same trend was observed with very low populations during identical periods to those observed on the first two crosses, a biological level between October 2010 and February 2011. In E. oleifera, by cons, no adult population of the insect was noted throughout the observation period, the evolution of the development cycle time being arrested at the stage of larvae with small individuals.

\subsection{Annual Fluctuation of Larvae, Pupae and Adults C. lameensis on Different Material}

In general, fluctuations in natural populations of $C$. $l a-$ meensis for all stage show distinct periods of proliferation. Between the years 2005 and 2011 it was noted a sawtooth evolution of the proliferation of C. lameensis. Between 2006 and 2007 the populations of pupae and adults were rare. By cons regarding larval populations, apart from 2007 where they remained low $(\approx 2$ individuals), they had oscillates irregularly between 43 in 2008 and 386 in the year 2011 during the observation period (Figure 4).

\subsection{Seasonal Fluctuation of Larval Populations of $C$. lameensis}

The largest populations of the pest have been observed during the short rainy season (248 larvae, 41 nymphs and 23 adults) (Figure 5). During the other seasons, the population of individuals of different stages is not half of the populations recorded during the short rainy season. 


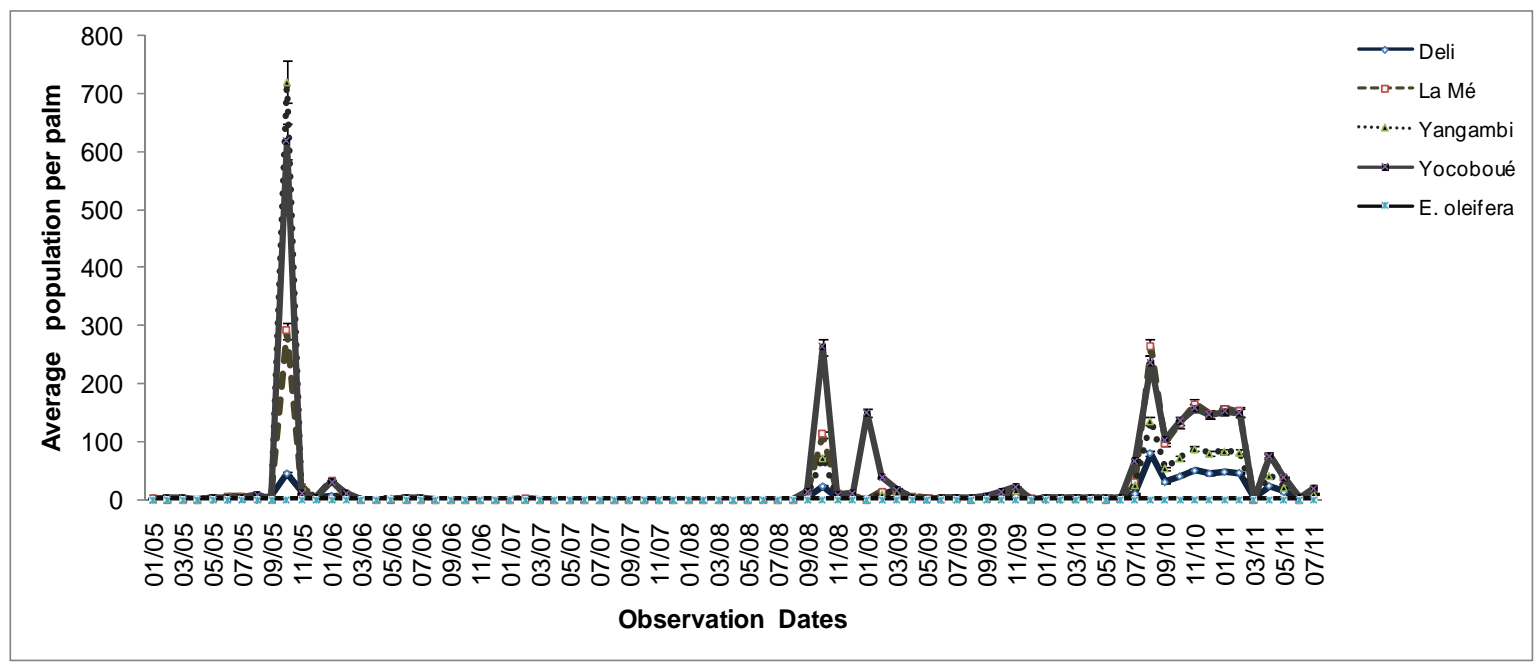

Figure 2. Dynamic of pupae’s populations of C. lameensis from 2005 to 2011.

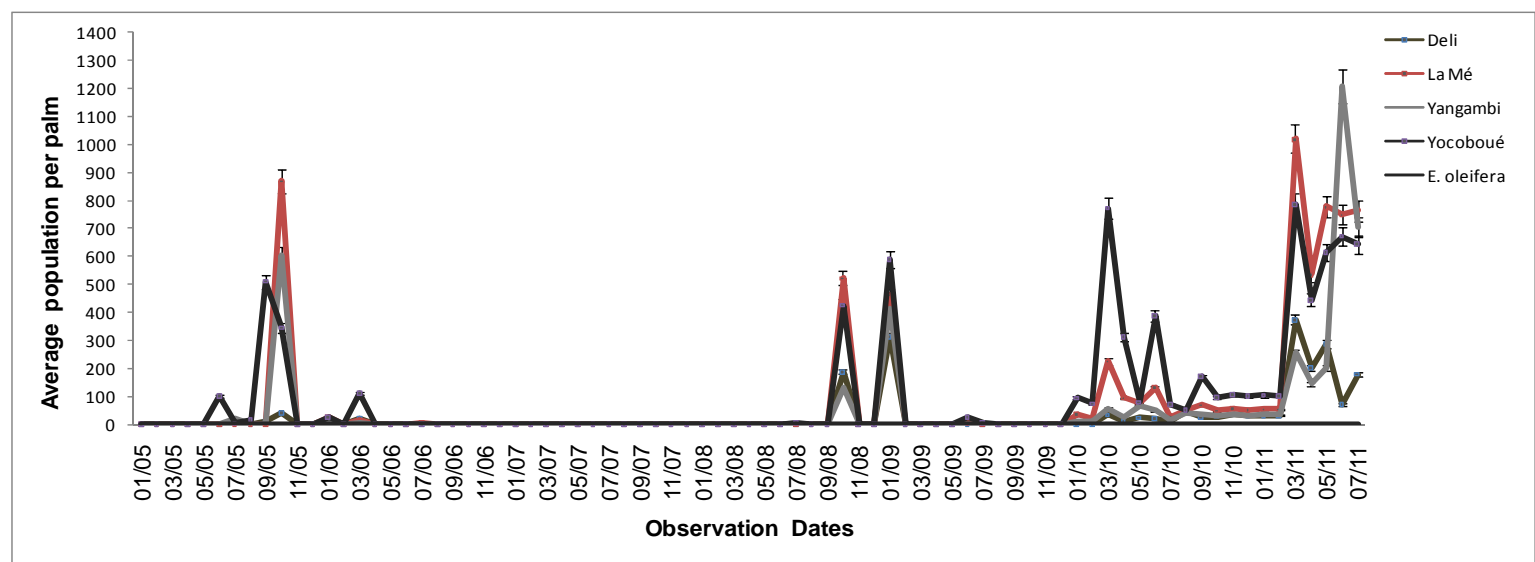

Figure 3. Dynamic of adult populations of C. lameensis from 2005 to 2011.

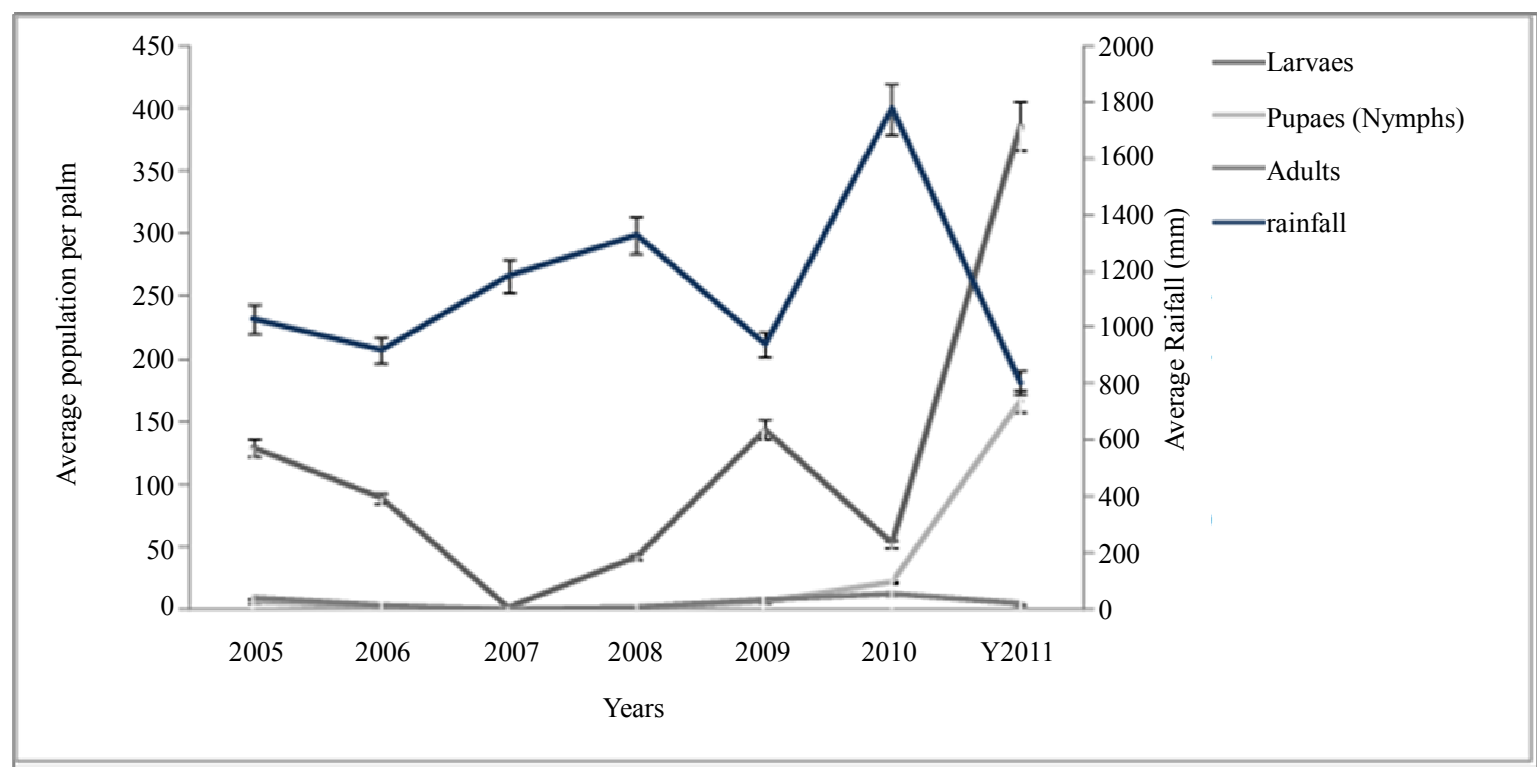

Figure 4. Dynamic of the populations of larvae, pupae and adults in time. 


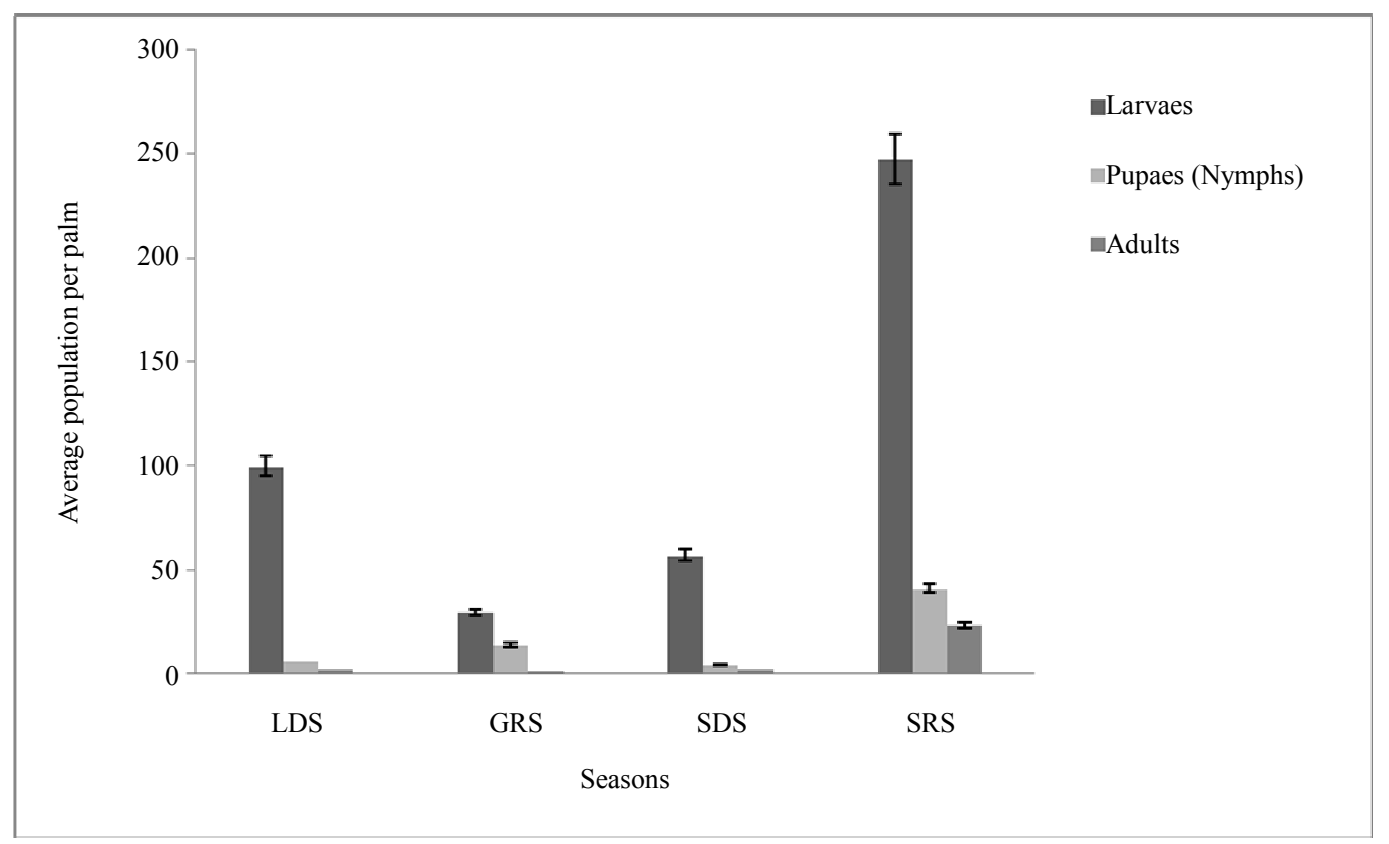

Figure 5. Population dynamics of larvae, pupae and adults of $C$. lameensis according to the season. LDS $=$ Long dry season (November-March); GRS = Great rainy season (March-August); SDS = Short dry season (August-mid-September); SRS = rainy season (mid-September-November).

\subsection{Fluctuation of $C$. lameensis Populations Depending on the Plant Material}

The average abundance of the population of $C$. lameensis as plant material (Figure 6) was more on the origins Yocoboué (215 larvae, 24 nymphs and 9 adults), followed by La Mé (115 larvae, 18 pupae and 5 adults), Yangambi (49 larvae, 10 nymphs and 5 adults) and Deli (40 larvae, 5 nymphs and 1 adult). In addition, oleifera were less attacked with very low larval populations $(<1$ individual). The stages of nymphs (pupae) and adults were not observed.

\section{Discussion}

The evolution of the natural population of the leaf miner (C. lameensis) depends on the oil palm species and the origin of the material in comparison. Thus, we note that the different development stages of insect is more evident on some crosses from Yocoboué where the average indices are higher in all larval, pupae, and adults stages in the early observations whereas on the other origins such as La Mé, Yangambi and Deli, despite the presence of these stages, the numbers recorded are lower. However, this infestation varies in these three origins and in terms of development stages, crosses from La Mé appear in second place, followed by Yangambi and Deli. As for E. oleifera they are much less or not attacked.

We note that the evolution of developmental stages (larvae and adults) takes place differently depending on the origins in comparison.

In addition, several peaks observed could result from the overlap of several generations of insects completing their life cycle within three months [6]. Climatic parameters play a role in the activity of parasitoid eggs. These climatic factors also influence fertility, lead to a decrease of the mortality of eggs, conditions for the genesis of outbreak.

The damage recorded on the palms of different materials reflects the distribution and size of populations of this pest. Therefore, there is a link between sensibility or tolerance of a cross to the level of damage caused on the material. Thus, the existence of favorable conditions or not during the life cycle of $C$. lameensis is certainly due to the intrinsic characteristics of origins, although abiotic and biotic conditions can play a significant role in the expression of this phenomenon.

Fluctuations in natural populations of $C$. lameensis for all stages, confirms two distinct periods of outbreaks in 2005 respectively, separated by 24 months from 2008 to 2009 during which the populations are virtually absent. Each outbreak lasts about a year. The population level of larvae during these outbreaks varies depending on the plant material. For both periods, there is a significant difference between the abundance of larval population of $C$. lameensis in the materials from Yocoboué and La Mé at one hand, Deli and Yangambi at the other. In 2005, populations vary over 1500 larvae per leaf on average at the beginning of the proliferation in Yocoboué origin before 


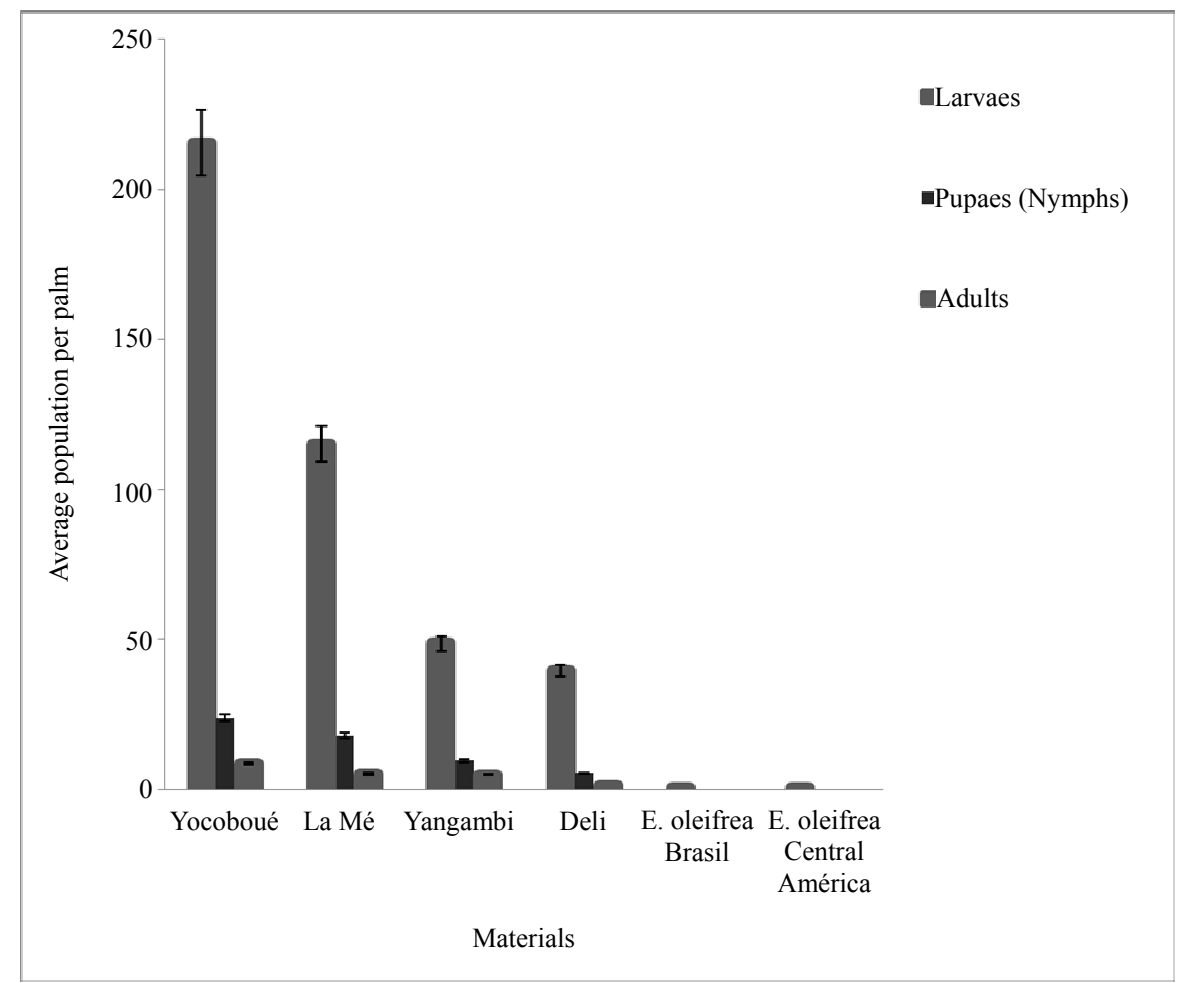

Figure 6. Population dynamics of larvae, nymphs and adults depending on the plant material.

falling and rising again to reach a plateau of more than 1000 individuals over several consecutive months before declining to after one year. The second outbreak in 2008 reveals larger population levels for the origins Yocoboué and La Mé up to about 3000 larvae per leaf on average. However, after 5 months, the populations' levels fall gradually and become economically acceptable after one year. The level of proliferation of populations of C. lameensis on E. Oleifera material for all stages (larvae, nymphs and adults) is insignificant in the past 5 years of observations. Only a few larvae are present at the time of the outbreaks in 2005 and 2008. No nymph or no adult is observed during outbreaks of the pest. This result suggests that the insect does not develop beyond the larval stages of the two origins of E. oleifera. The level of the larval population is significantly important during the short rainy season (180 larvae on average) and to a lesser extent during the long dry season (80 larvae on average).

\section{Conclusion}

The level of natural populations is characterized by periods of heavy outbreaks that last for almost a year during which a peak of 3000 larvae per leaf in averages was noted following a period of latency lasting two years with weak number or no larvae. The origins of West Africa (Yocoboué and La Mé) were significantly more affected than the origins of Central Africa and Asia (Yan- gambi and Deli). Populations grow during the short rainy season which is favorable for the hatching. The development of larval stages continues during the short rainy season. The evolution of developmental stages (larvae, nymphs and adults) takes place differently in the four origins of E. guineenis and two origins of E. oleifera in comparison. Thus, we can classify them in the order of increasing intensity of pest attacks: E. oleifera, E. guineensis: Deli, Yangambi, La Mé and Yocoboué.

\section{Acknowledgements}

The authors express their gratitude to the authorities of the Centre de Recherches Agricoles Plantes Pérennes de Pobè of Institut National des Recherches Agricoles du Bénin, the PalmElit and International Cooperation Centre in Agronomic Research for Development for their technical and financial support for the research.

\section{REFERENCES}

[1] G. W. Chang, H.-W. Lin and S.-K. Chen, "Modeling Characteristics of Harmonic Currents Generated by HighSpeed Railway Traction Drive Converters," IEEE Transactions on Power Delivery, Vol. 19, No. 2, 2004, pp. 766773. doi:10.1109/TPWRD.2003.822950

[2] J. L. Jacquemard, "The Oil Palm," In: The Technician of Tropical Agriculture No. 33 and Maisonneuve Editions, Larousse, Paris, 1995, pp. 165-172. 
[3] N. Berti and D. Mariau, "Coelaenomenodera lameensis n. sp. Pest of Oil Palm. Entomological," Nouvelle Revue, Vol. 16, 2000, pp. 253-267.

[4] P. Cachan, "The Hispidae Miner (Coelaenomenodera elaeidis MLK), Oil Palm Pest in the Guinean Zone," Tropical Agronomy, Vol. 12, 1957, pp. 610-632.

[5] Morin and Mariau, "Etude sur la Biologie de Coelaenomenodera elaeidis. Morphologie et Etude du Développement," Oléagineux, Vol. 25, No. 1, 1970, pp. 11-16.

[6] D. Mariau and J. P. Besombes, "Method of Controlling Population Levels of Coelaenomenodera lameensis," Oilseeds, Vol. 27, 1972, pp. 425-427.

[7] R. Philippe, "Practical Methods of Chemical Control against Coelaenomenodera minuta Uhrmann (Coleoptera: Chrysomelidae-Hispinae)," Oilseeds, Vol. 45, 1990, pp. 165-172.

[8] R. Philippe, "Visit to the Centre for Agricultural Research Perennial Plants (CRA-PP) Benin Pobè Station," Mission Report, 2003.

[9] Berti and Mariau, "Coelaenomenodera lameensis n. sp. Pest of Oil Palm," Nouvelle Revue, Vol. 16, 2000, pp. 253-267.

[10] R. Philippe, "Study of the Development of Coelaenome- nodera elaeidis Mlk (Coleoptera: Chrysomelidae-Hispinae) on Hybrid E. guineensisx E. melanococca," Oilseeds, Vol. 32, 1977, pp. 1-4.

[11] A Coffi, "Study of the Development of the Leaf Miner, $C$. lameensis Two Backcross of Interspecific Hybrid Origins and Four Palm Oil," 2006.

[12] A. Coffi, P. Atachi and R. Philippe, "Development of Coelaenomenodera lameensis Berti (Coleoptera: Chrysomelidae-Hispinae) on Various Sources of Oil Palm," Annals of Agricultural Sciences of Benin, Vol. 12, No. 1, 2009, pp. 1-14.

[13] A. Coffi, "Report of the End of the Operation against Coelaenomenodera lameensis," Centre for Agricultural Research Perennial Plants (CRA-PP), Pobè Benin Station, 2000.

[14] Mariau and Morin, "The Biology of Coelaenomenodera elaeidis MLK, the Natural Larvae Mortality," Oléagineux, Vol. 29, No. 12, 1974, pp. 551-555.

[15] S. Manel and D. Debouzie, "Logistic Regression and Continuation Ratio Models to Estimate Insect Development under Variable Temperatures," Ecological Modelling, Vol. 98, No. 2-3, 1997, pp. 237-243. doi:10.1016/S0304-3800(96)01908-4 